\title{
Electrowetting-Induced Oil Film Entrapment and Instability
}

\author{
Adrian Staicu and Frieder Mugele* \\ Physics of Complex Fluids, Faculty of Science and Technology, University of Twente, \\ P. O. Box 217, 7500AE Enschede, The Netherlands \\ (Received 12 May 2006; published 16 October 2006)
}

\begin{abstract}
We investigate the spreading at variable rate of a water drop on a smooth hydrophobic substrate in an ambient oil bath driven by electrowetting. We find that a thin film of oil is entrapped under the drop. Its thickness is described by an extension of the Landau-Levich law of dip coating that includes the electrostatic pressure contribution. Once trapped, the thin film becomes unstable under the competing effects of the electrostatic pressure and surface tension and dewets into microscopic droplets, in agreement with a linear stability analysis. Our results recommend electrowetting as an efficient experimental approach to the fundamental problem of dynamic wetting in the presence of a tunable substrate-liquid interaction.
\end{abstract}

DOI: 10.1103/PhysRevLett.97.167801

Electrowetting $(\mathrm{EW})$ is a classic example of interaction between fluids and electric fields: by applying a voltage between a drop of conductive liquid and an insulatorcovered hydrophobic electrode, the contact angle $\theta$ of the drop can be reduced by several tens of degrees compared to Young's angle [1]. The efficiency, reproducibility and ease of use make electrowetting preferable over other manipulating methods for small liquid drops, such as Marangoni or thermocapillary stresses [2] or switchable selfassembled monolayers. Hence electrowetting is increasingly becoming the actuation method for open digital microfluidic devices ([1] and references therein). Apart from technological applications, EW has also proven to be a very useful tool for studying fundamental problems in wetting and thin film hydrodynamics, where the contact angle is often a crucial parameter that is difficult if not impossible to vary experimentally without changing other important aspects of the system. Examples include wetting of complex surfaces [3,4], capillary pinch-off and microdroplet generation [5-7], and deposition [8]. Frequently, electrowetting experiments are performed in an ambient oil bath in order to minimize both the evaporation of liquid and contact angle hysteresis. It has been indicated by several authors [9-13] that thin layers of the ambient oil might form between the drop and substrate in such a twophase configuration. Quilliet and Berge [9] found theoretically that the balance between electrical forces and the disjoining pressure should give rise to an equilibrium thickness of the films of approximately $10-20 \mathrm{~nm}$ for typical values of the applied voltage. However, despite the importance of these layers - for instance for the reduction of contact angle hysteresis, but also for the protection of the surfaces from adsorption of biomolecules $[10,14]-$ their properties and formation mechanism remained elusive in previous experimental studies [11].

In the present Letter we study the dynamics of moving contact lines in EW systems with a two-phase configuration, as just described. We show that a layer of oil is indeed entrapped under the drop with an initial thickness that is determined by the hydrodynamics of the moving contact
PACS numbers: 68.15.+e, 68.05.-n, 83.60.Np

line rather than by equilibrium properties. To describe the entrapment process we extend the Landau-Levich [15] treatment of dynamic wetting by an additional electrostatic pressure contribution, a topic that attracted considerable attention in the recent wetting literature [16-18]. Following the entrapment, the oil film turns out to be unstable and breaks up into a number of smaller oil droplets. The size distribution of these droplets is described by a linear stability analysis of the thin film in the lubrication approximation, taking into account the balance between surface tension and electrostatic pressure. The problem thus combines two aspects: the entrapment process itself and the subsequent time evolution of the entrapped film.

The experimental setup is an adaptation of the classic EW configuration [1]: a microliter water drop (with added $\mathrm{NaCl}$ for enhanced electrical conductivity) sits on a coated glass substrate, as sketched in Fig. 1. The coating consists of two layers: electrically conducting ITO (indium tin oxide) and a hydrophobic Teflon AF (DuPont) coating with a thickness of $d=0.8 \mu \mathrm{m}$, which serves as dielectric. A gold electrode connects the water drop to a power supply of $\mathrm{dc}$ or $10 \mathrm{kHz}$ ac voltage and the circuit is closed

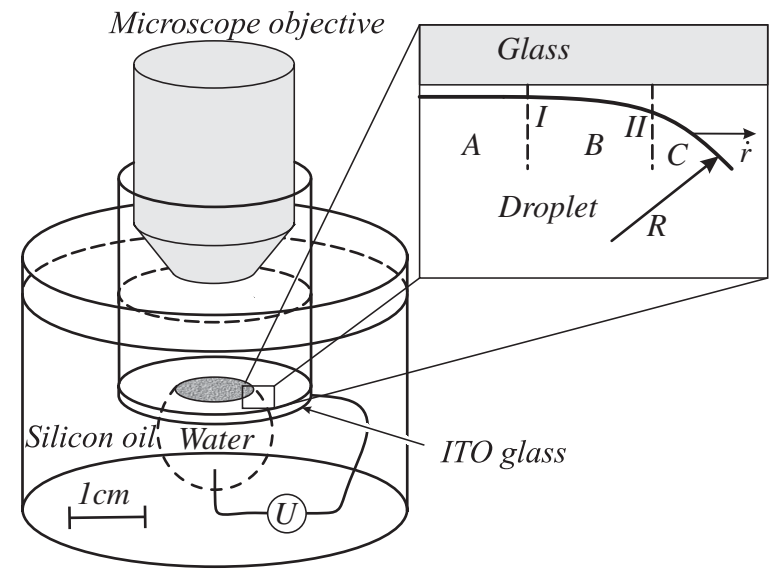

FIG. 1. Sketch of the electrowetting setup. The dimensions of the water droplet are exaggerated. 
through the ITO substrate. The entire system is immersed in silicone oil (Fluka AS100 with viscosity $\mu \approx$ $100 \mathrm{mPas}$ ) and observed through the $10 \times$ magnification objective of an interference microscope. The drop size is chosen such that the radius $R_{0} \sim 1 \mathrm{~mm} \ll l_{c}=$ $\sqrt{\gamma /(g \delta \rho)}$, where $l_{c}$ is the capillary length and $\delta \rho$ the oil-water density difference ( $g$ : gravitational acceleration). When a fixed voltage $U$ is applied, the drop achieves equilibrium with an apparent contact angle that obeys the EW equation [1]:

$$
\cos \theta=\cos \theta_{Y}+\frac{\varepsilon_{0} \varepsilon_{d}}{2 d \gamma} U^{2}=\cos \theta_{Y}+\eta,
$$

where $\varepsilon_{d}$ is the dielectric constant of Teflon AF (catalog value $\varepsilon_{d} \approx 2$ ), $\gamma=34 \mathrm{~mJ} / \mathrm{m}^{2}$ the oil-water interfacial tension and $\eta$ the electrowetting number. The Teflon coating provides a Young angle $\theta_{Y}$ close to $180^{\circ}$. The maximum voltage $U_{\max }=25 \mathrm{~V}$ in the present experiments was chosen sufficiently small to avoid contact angle saturation effects [1] or damage of the dielectric layer, which occurs only at a field strength of $\approx 200 \mathrm{~V} / \mu \mathrm{m}$ in our system. The minimum contact angle is $\theta\left(U_{\max }\right) \approx 145^{\circ}$.

The experiments are performed as follows: initially, at time $t=0$, a spherical drop is positioned at zero voltage at a distance of a few micrometers below the substrate. Subsequently, we increase the voltage linearly in time with ramp speeds $d U / d t$ ranging from 0.25 to $25 \mathrm{~V} / \mathrm{s}$. Upon doing so, the drop approaches the surface and spreads with continuously decreasing $\theta$ following Eq. (1). The combination of volume conservation, Eq. (1), and the linear voltage ramp gives rise to an (essentially) linear increase of the radius $r(t) \sim t \sim U$ [19] of the substrate-drop interface. As the drop spreads interference fringes appear (see Fig. 2) indicating that a film of the ambient oil with a thickness of the order of the wavelength of visible light is entrapped under the drop. Only close to the advancing three phase contact line (TCL) the film surface is smooth (Fig. 2: $t=0.1$ and $t=0.2 \mathrm{sec}$ ). As the drop spreads further the oil film displays thickness modulations and eventually breaks up into small droplets. The time it takes for the instability to grow into droplets ranges from a few tenths of a second to minutes, increasing with decreasing ramp speed and increasing radial position. In the final state (when the voltage is kept at $U_{\max }=25 \mathrm{~V}$ ), the film breaks up into an azimuthally symmetric pattern of microscopic droplets (Fig. 2). Their sizes decrease approximately inversely proportional to the distance $r$ from the center (4). We repeated the experiments at fixed ramp speed and verified that the film breakup leads to different random droplet arrangements of similar radial distribution, thus excluding the possibility of surface defects as driving force for the breakup. Similar patterns are obtained for all voltage ramp speeds. It is important to note that even for the slowest ramps the droplets remain visible with lateral diameters on the order of $10 \mu \mathrm{m}$. From the observations described so far, we conclude that we can split the dynamics of the system into two separate parts, namely, the

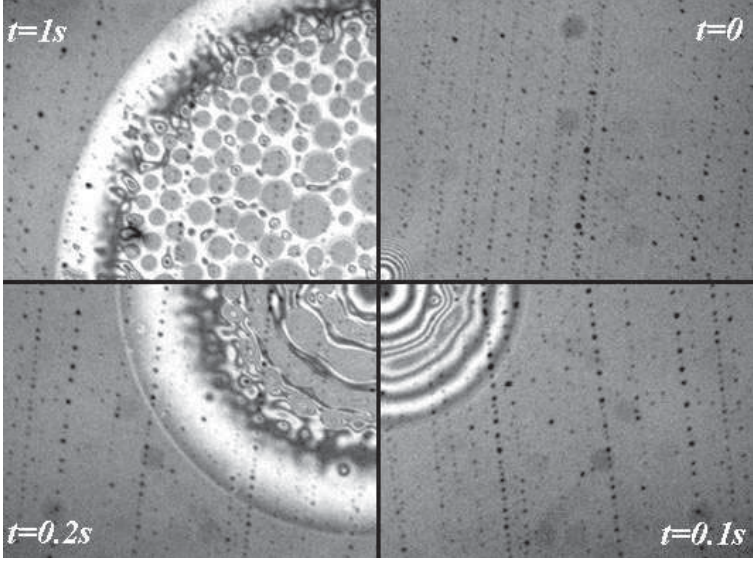

FIG. 2. Microscope images taken at the substrate-droplet interface during the application of a fast voltage $\operatorname{ramp} d U / d t=$ $25 \mathrm{~V} / \mathrm{s}$. The thin oil film trapped under the water drop destabilizes progressively and breaks up into microscopic droplets. The tiny dark condensation spots are situated on the opposite side of the glass substrate.

hydrodynamic entrapment of the oil film and its subsequent instability.

Let us first analyze the stability of an already entrapped oil film with a constant thickness $e$. The free energy $F_{U}(e)$ of such a film depends on the interfacial energies $\gamma$ of the oil-water and $\gamma_{\mathrm{os}}$ of the oil-substrate interface and on the electrostatic energy in the system. If we include the van der Waals interaction with a Hamaker constant $A$ for the sake of completeness, we have $[9,20]$

$$
F_{U}(e)=\gamma_{\mathrm{os}}+\gamma+\frac{A}{12 \pi e^{2}}-\frac{\varepsilon_{0} \varepsilon_{d} U^{2}}{2 d\left(1+\frac{e \varepsilon_{d}}{d \varepsilon_{\mathrm{oil}}}\right)},
$$

where the last term is simply the electrostatic energy of a parallel plate capacitor filled with two layers of different dielectrics parallel to the substrate. While the van der Waals term may either stabilize or destabilize a thin film, depending on the sign of $A$ [9], the electrostatic term is always destabilizing.

To understand the mechanism of droplet formation and ultimately the size distribution of the droplets, we employ standard linear stability analysis in lubrication approximation [21] in one dimension denoted by $x$ for the free energy given in Eq. (2). As usual, the analysis is valid for small variations $u(x) \ll e$ of the film thickness with $|\nabla u| \ll 1$. We solve the thin film equations for the time evolution of modulations of the general form $u(x, t)=e+u_{0} e^{i q x} e^{t / \tau}$ [21] and determine the fastest growing mode by finding the minimum of $\tau$. In Fig. 3 we plot the wavelength of this mode

$$
\lambda_{m}=2 \sqrt{2} \pi \gamma^{1 / 2}\left(-\frac{A}{2 \pi e^{4}}+\frac{\varepsilon_{0} \varepsilon_{d}^{3} U^{2}}{d^{3}\left(1+\frac{e \varepsilon_{d}}{d \varepsilon_{\mathrm{oil}}}\right)^{3} \varepsilon_{\mathrm{oil}}^{2}}\right)^{-1 / 2}
$$

versus $e$ for several values of $U$ and for a negative $A=$ $-1.83 \times 10^{-21} \mathrm{~J}$, as derived from the refractive indices of the materials in our system using textbook equations [22]. 


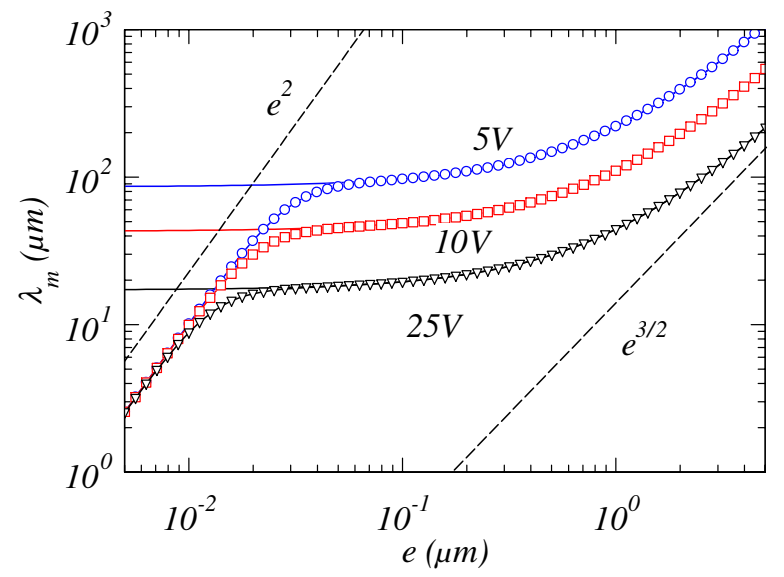

FIG. 3 (color online). Dependence of fastest unstable wavelengths $\lambda_{m}$ (open symbols) on the initial thickness of the oil film $e$ for several voltages $U$. Solid lines: electrostatic contribution only: $A=0$.

From Eq. (3), it is obvious that $\lambda_{m}$ depends on both $U$ and $e$. For very thin films, $e<\approx 20 \mathrm{~nm}$, the characteristic $\lambda_{m} \sim e^{2}$ behavior due to van der Waals interaction becomes apparent. At larger film thickness (i.e., in the range of interest here - as we concluded from the appearance of interference fringes in Fig. 2), however, the electrostatic term dominates. While the latter gives rise to a $\lambda_{m} \sim e^{3 / 2}$ behavior at very large $e$, the finite thickness $d$ of the insulating layer gives rise to a plateau for $e$ between a few tens of nanometers and almost $1 \mu \mathrm{m}$.

Let us now compare the experimental droplet diameter distribution with $\lambda_{m}$ from Eq. (3), which we assume to be the same up to a constant of order unity. Given the weak dependence of $\lambda_{m}$ on $e$ within the range of interest, we assume for the moment a constant thickness of the entrapped film of, say, $e=400 \mathrm{~nm}$. Under these conditions the radial dependence of the droplet size simply reflects the fact that larger radii are reached later in time while we are ramping up the voltage, i.e., at a higher voltage. Since $U \sim r$ (see above), we expect indeed $\lambda_{m} \sim$ $r^{-1}$ from Eq. (3), in reasonable agreement with the experimental data (see Fig. 4). The agreement confirms our implicit assumption that the selection of the fastest growing mode at $r$ occurs essentially immediately after the TCL passed that position.

To confirm the model further, we compared the droplet sizes obtained at a fixed radial position (i.e., at fixed voltage) for various ramp speeds. As we will confirm below, we expect intuitively the film thickness to be thinner for slower ramps because slower speeds allow more time for squeezing out the entrapped oil. In Table I we compare the droplet diameters, extracted at $r=125 \mu \mathrm{m}$, at different $d U / d t$. While the speed of the TCL varies by a factor of $10^{2}$, the average diameter of the droplets changes only by a factor of $\approx 2$. This corroborates that the experimentally observed values of $\lambda_{m}$ correspond to the "plateau" region of Fig. 3.

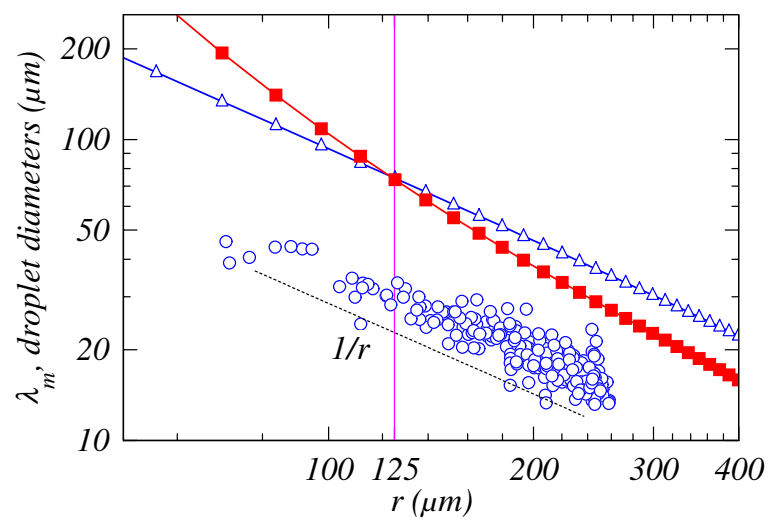

FIG. 4 (color online). (○) Measured radial dependence of oil droplet diameters for $d U / d t=25 \mathrm{~V} / \mathrm{s}$ and ( $\mathbf{\square})$ prediction of linear stability analysis using either measured film thicknesses $e(r)$ (from Fig. 5) or constant $e=400 \mathrm{~nm}(\triangle)$.

We now turn to the dynamics of oil film entrapment. A direct measurement of film thickness prior to breakup is possible by analyzing the interference patterns which are (briefly) visible close to the TCL before the onset of the instability (inset of Fig. 5). To obtain a quantitative value for the film thickness, we calculated the reflectance of a Teflon-oil-water layered structure for normal incident light as a function of the oil film thickness $e$ [23]. We found that the extracted intensity profiles could be fitted by an algebraic test function $e(r)=a / r^{b}$, with $b=1.35 \pm$ 0.1 . To check the consistency of the linear stability analysis, we inserted the fitted thickness profiles $e(r)$ into Eq. (3) and calculated $\lambda_{m}(r)$. The results are shown in Fig. $4(\triangle$ symbols) and in Table I. To a fair degree of accuracy, we find that the ratio between $\lambda_{m}$ and the droplet diameter is between 2 and 3 .

A particularly interesting aspect, however, is the power law behavior of $e(r)$. The motion of the TCL is similar to the film entrainment problem in dip coating. In the classical analysis of Landau and Levich [15], the thickness of the entrapped films follows the law $e \sim \mathrm{Ca}^{2 / 3}$, valid for small capillary numbers $\mathrm{Ca}=\mu \dot{r} / \gamma \ll 1$. This law is derived by balancing the viscous dissipation $\mu \dot{r} / e^{2}$ and capillary pressure near the TCL (see close-up of this region in Fig. 1). As the TCL moves with uniform velocity $\dot{r} \sim$ $d U / d t$ in our experiments, the capillary number is fixed for each voltage ramp and one expects the entrapped film to be flat. Regions $A$ (where the entrapped film of thickness

TABLE I. Average droplet size against the ramp speed $d U / d t$. The values are measured at fixed location $r=125 \mu \mathrm{m}$. The last row shows the calculated unstable wavelengths $\lambda_{m}$ [Eq. (3)] for film thicknesses $e$ estimated from interference patterns Fig. 5 with the use of Eq. (3).

\begin{tabular}{lcccccccc}
\hline \hline$d U / d t(\mathrm{~V} / \mathrm{s})$ & 0.25 & 0.5 & 1 & 1.67 & 2.5 & 5 & 12.5 & 25 \\
\hline Droplet size $(\mu \mathrm{m})$ & 16 & 16 & 17 & 18 & 20 & 23.4 & 26.6 & 33.5 \\
$\lambda_{m}(\mu \mathrm{m})$ & - & 50.3 & - & 52.7 & - & 56.1 & - & 73.7 \\
\hline \hline
\end{tabular}




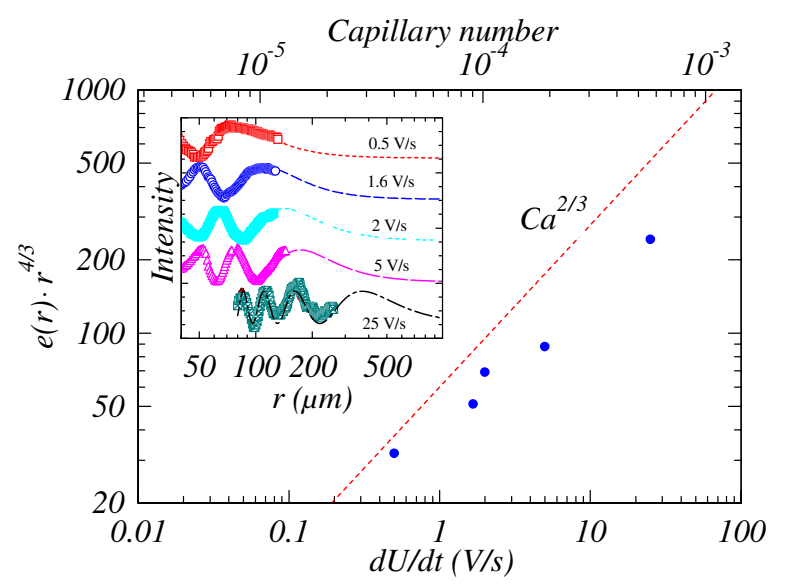

FIG. 5 (color online). Film thickness (compensated by the electrowetting contribution $r^{4 / 3}$ ) against the capillary number ( $r$ is given in $\mu \mathrm{m}$ ). Inset: interference fringe intensity fitted with the power law $e(r)=a / r^{4 / 3}$.

$e$ is flat) and $C$ (moving meniscus with constant curvature $2 / R$ ) are separated by a dynamic meniscus (region $B$ ) of length $l$ where most of the viscous dissipation takes place. The pressure gradient driving the flow is then approximately $\Delta p / l=\left(p_{R}^{I I}-p_{R}^{I}\right) / l=\left(\frac{2 \gamma}{R}-0\right) / l$. The length $l$ is obtained by asymptotically matching the curvatures of the "static" and dynamic menisci in the small-slope approximation, leading to $l=\sqrt{R e}$. Inserting this into the force balance results in the Landau-Levich law mentioned above. In the presence of $\mathrm{EW}$, however, the interface potential [Eq. (2)] gives rise to an additional pressure $p_{\mathrm{el}}=$ $\frac{d F_{U}}{d e}$ which is finite at position I but negligible at II [24]. To a good approximation the pressure gradient then becomes $\Delta p / l=\left(p_{\mathrm{el}}^{I}+p_{R}^{I I}\right) / l=\left(\frac{\varepsilon_{0} \varepsilon_{d}^{2}}{2 d^{2} \varepsilon_{\mathrm{oil}}} U^{2}+\frac{\gamma}{R}\right) / l \approx \frac{\gamma}{l d} \eta \frac{\varepsilon_{d}}{\varepsilon_{\mathrm{oil}}}$. As a result we obtain a modified Landau-Levich law

$$
e(r) \sim(d \sqrt{R})^{2 / 3}\left(\frac{\mathrm{Ca}}{\eta}\right)^{2 / 3} \sim \frac{\mathrm{Ca}^{2 / 3}}{U^{4 / 3}} \sim \frac{\mathrm{Ca}^{2 / 3}}{r^{4 / 3}} .
$$

Therefore, the influence of the electrowetting number $\eta$ manifests itself in the radial dependence of the film thickness $e(r) \sim r^{-4 / 3}$, which is in good agreement with the measured exponents $b$. In Fig. 5 we isolated the "pure" capillary number dependence by compensating on the electrowetting contribution. [This is accomplished by using single parameter fits $e(r)=a / r^{4 / 3}$ for the film thickness.] Clearly, $e$ depends on $\mathrm{Ca}$ with an exponent close to $2 / 3$. A full treatment of the problem along the lines of $[16,18]$ should account for these deviations. Such a treatment will also have to take into account the field-induced distortions of the equilibrium profile that we reported earlier [25]. By varying the thickness of the dielectric layer, we expect to be able to tune the relative importance of the electrostatic contribution in future experiments.

We thank Rob Hayes (Philips Research) for providing substrates of outstanding surface quality. Financial support within the joint program Micro- and Nanofluidics by the $\mathrm{MESA}_{+}$institute for nanotechnology and by the IMPACT institute at Twente University is gratefully acknowledged.

*Electronic address: a.d.staicu@ tnw.utwente.nl

[1] F. Mugele and J.-C. Baret, J. Phys. Condens. Matter 17, R705 (2005).

[2] A. Darhuber and S. Troian, Annu. Rev. Fluid Mech. 37, 425 (2005).

[3] J.-C. Baret, M. Decŕe, S. Herminghaus, and R. Seemann, Langmuir 21, 12218 (2005).

[4] T. Krupenkin, J. Taylor, T. Schneider, and S. Yang, Langmuir 20, 3824 (2004).

[5] M. Vallet, M. Vallade, and B. Berge, Eur. Phys. J. B 11, 583 (1999).

[6] F. Mugele and S. Herminghaus, Appl. Phys. Lett. 81, 2303 (2002).

[7] J.-C. Baret and F. Mugele, Phys. Rev. Lett. 96, 016106 (2006).

[8] J.-C. Baret and M. Brinkmann, Phys. Rev. Lett. 96, 146106 (2006).

[9] C. Quilliet and B. Berge, Europhys. Lett. 60, 99 (2002).

[10] V. Srinivasan, V. Pamula, and R. Fair, Lab Chip 4, 310 (2004).

[11] M. Bienia, F. Mugele, C. Quilliet, and P. Ballet, Physica (Amsterdam) A339, 72 (2004).

[12] M. Bienia, Ph.D. thesis, Universite Joseph Fourier Grenoble, 2005.

[13] R. Hayes (private communication).

[14] F. Saeki, J. Baum, H. Moon, J. Yoon, C. Kim, and R. Garrell, Polym. Mater. Sci. Eng. 85, 12 (2001).

[15] V. Levich, Physicochemical Hydrodynamics (PrenticeHall, N.J., 1962).

[16] R. Krechetnikov and G. Homsy, Phys. Fluids 17, 102105 (2005).

[17] J. Eggers and H. Stone, J. Fluid Mech. 505, 309 (2004).

[18] G. F. Teletzke, H. T. Davis, and L.E. Scriven, J. Phys. (Paris) 23, 989 (1988).

[19] If $R_{0}$ is the initial radius of the undeformed drop, then at $U \neq 0$ the drop volume is $\pi R(U)^{3}\left[\frac{2}{3}-\frac{3}{4} \cos \theta(U)+\frac{1}{12} \times\right.$ $\cos 3 \theta(U)]=\frac{4 \pi R_{0}^{3}}{3}[R(U)$ is the voltage-dependent radius]. The radius of the oil film is then $r=R \sin \theta$. The error made by ignoring the oil layer in the EW curve is $\delta r / r \leq$ $4 \%$ for an oil film of thickness $e=0.1 \mu \mathrm{m}$.

[20] S. Herminghaus, Phys. Rev. Lett. 83, 2359 (1999).

[21] P. de Gennes, F. Brochard-Wyart, and D. Quere, Capillarity and Wetting Phenomena: Drops, Bubbles, Pearls, Waves (Springer, New York, 2004).

[22] J. Israelachvili, Intermolecular and Surface Forces (Academic, London, 1992).

[23] M. Born and E. Wolf, Principles of Optics (Cambridge University Press, Cambridge, England, 1999).

[24] Asymptotic matching of curvatures occurs in a region where the film thickness is very large compared to that of the entrapped film $e_{I}$ [15]. Since, $p_{\mathrm{el}}$ decreases approximately like $1 / e^{2}$, we have $p_{\mathrm{el}}^{I I} \ll p_{\mathrm{el}}^{I}$.

[25] J. Buehrle, S. Herminghaus, and F. Mugele, Phys. Rev. Lett. 91, 086101 (2003). 